\title{
GRADER: A computer program that recommends student letter grades
}

\author{
MICHAEL E. MILLS \\ Loyola Marymount University, Los Angeles, California
}

\begin{abstract}
GRADER, a computer program that makes student test and/or course letter grade recommendations to instructors, is described. The program uses an algorithm to combine the content mastery (percentage correct) and peer comparison ("curve") approaches to set grade cutoffs. In addition to standardizing the procedures with which letter grade cutoffs are made, GRADER may help to reduce instructor grading subjectivity and obviate tendencies toward grade inflation or deflation.
\end{abstract}

Many problems are associated with current methods of determining test and course letter grade cutoffs (Bresee, 1976; Cureton, 1971; Hanna \& Cashin, 1988; Popham, 1978). As Dressel (1983, p. 12) asserted, a grade is "an inadequate report of an inaccurate judgment by a biased and variable judge of the extent to which a student has attained an undefined level of mastery of an unknown proportion of an indefinite material."

Some of the most serious problems associated with many current methods of determining letter grade cutoffs include: (1) subjectivity, (2) the consequent lack of standardization of grading practices between courses and professors, and (3) the tendency toward grade inflation (or deflation) by some instructors. The latter two concerns cannot be adequately addressed, I believe, until the subjectivity of establishing grade cutoffs is reduced.

There are two popular, and very different, methods of determining grade cutoffs: content mastery and peer comparison grading. Each method of determining grades reflects a different educational philosophy. Those who prefer content mastery grading presume that the "percentage correct score" is the appropriate index of student performance. In contrast, peer comparison graders believe that the "percentile score" (the percentage of students with a lower test score) is a more appropriate measure of student performance. (A third educational philosophy, "mastery learning," typically does not result in grade assignments; thus this approach will not be discussed here.)

Content mastery grading involves "absolute" measurement; peer comparison grading consists of comparative, or ipsative, measurement. Ratings assume an interval level of measurement but may be severely distorted by response biases and styles; rankings only achieve ordinal measurement (and thus may exclude information), and can force an indication of difference where no appreciable difference may exist (Bauemfeind, 1962; Hicks, 1970). Thus, content mastery and peer comparison grading methods are based on the evaluation of very different types of information.

Correspondence should be addressed to Michael E. Mills, Department of Psychology, 7101 W. 80th St., Loyola Marymount University, Los Angeles, CA 90045 .
We should acknowledge that the issue of how best to determine grade cutoffs is a knotty measurement problem. It is unfortunate that instructors have been left to grapple with such complex statistical issues without either guidelines developed by measurement specialists or assistance provided by computerized "expert systems."

\section{CONTENT MASTERY GRADING}

Instructors who prefer this method of grading believe that performance should be evaluated by reference to student mastery of the material, typically in terms of a percentage correct score. The traditional percentage correct grade cutoff is familiar: $90 \%=\mathrm{A}, 80 \%=\mathrm{B}, 70 \%=\mathrm{C}$, etc. Note that, with content mastery grading, it is entirely possible for all the students in a class to get As. It is also entirely possible for all the students in a class to get Fs. In that sense, every student's performance is completely independent of the performance of other students. Content mastery grading is an example of what psychometricians call "absolute" or "independent" assessment. The pros and cons of this method of set grade cutoffs are listed below.

\section{Advantages}

1. Students are not in competition with one another for course grades when this method is used, which may encourage cooperation between students to master the material. They may form cooperative study groups and share course materials.

2. Grade cutoffs can be made known to students at the outset of the class. There is less inducement to dispute the fairness of those cutoff points later (although the fairness of tests may still be disputed).

3. If an entire class performs well, all students may be appropriately rewarded. Because there is no forced distribution of grades, no one need receive a lower grade than he or she has earned.

\section{Disadvantages}

1. Content mastery grading may make grade cutoffs explicit, but that in itself does not ensure that the cutoff 
points are in any sense fair. Tests vary in their overall difficulty and fairness. A poorly constructed test may not have content validity (i.e., it may not be a representative sample of the material that students are responsible for learning).

2. Not only does content mastery grading fail to account for the overall difficulty level or fairness of the test, it does not factor in an individual student's performance relative to the performance of other students in the class. There is no extra reward for being the top performer in the class, and no extra penalty for being at the bottom of the class.

3. Because no particular grade distribution is required, this method may leave grade inflation unchecked.

\section{PEER COMPARISON GRADING}

Instructors who prefer this method of grading believe that student performance should be evaluated through reference to the performance of the class as a whole, typically through reference to each student's percentile score. This method is traditionally called "grading on a curve." It is based on the generally valid psychometric assumption that when there is variation in a human trait, that variation is normally distributed. Grading on the curve thus represents grading on a "normal" curve, wherein equal percentages of students (say 7\%) obtain As and Fs, and equal percentages (say $24 \%$ ) receive Bs and Ds. The remaining 38\% receive Cs. An alternative and less stringent set of cutoffs is suggested by Kaplan and Saccuzzo (1982): the 85th percentile or above is an A; between the 60th and 84th percentiles is a B; between the 20th and 59 th percentiles is a $C$; between the 6 th and 19 th percentiles is a D; the 5th percentile and below is an F.

Note that each student's grades are completely dependent on the student's performance relative to that of peers. Peer comparison grading is an example of what psychometricians call ipsative, nonindependent, or comparative assessment (Hicks, 1970).

\section{Advantages}

1. It is true that the variation in most human traits can be described well by the normal curve. Grading on a normal curve may indeed adequately represent the underlying distribution of mastery of course material much of the time.

2. The world outside of academia is competitive. A competitive grading system may help students learn to cope effectively in competitive situations.

3. The peer comparison approach forces grading discipline on faculty, making grade inflation impossible.

4. If instruction, tests, or the textbook are below par, this method of grading will help control for such variability. The same percentages of students will still get each letter grade, even if the class as a whole performs very poorly on difficult examinations.

5. Peer comparison grading may discourage collaborative cheating on exams because all students are in competition with one another.

\section{Disadvantages}

1. At times, peer comparison grading results in unfair grade assignments. The difference between a $\mathrm{C}$ and an F may be as little as a few test points, given a highly skewed distribution. This is because an interval level of measurement is not achieved-only ordinal distinctions between students are made. The resulting grades thus may not accurately represent underlying differences in mastery of the course material.

2. Because students are in competition with one another, they may not study cooperatively. During times of severe competition, students may actually attempt to sabotage one another's experiments or assignments.

3. Because an interval level of measurement is not achieved, it is not possible to perform common statistical analyses that require the assumption of equal intervals between measurement points.

\section{COMBINED APPROACHES TO DETERMINING GRADE CUTOFFS}

Although many instructors have made a choice between the two grading methods above, some may feel vaguely uncomfortable with their decision. Both content mastery and peer comparison methods of determining grade cutoffs have their respective assets and liabilities. I suspect that this discomfort has led many faculty, if not most, to attempt to combine the peer comparison and content mastery methods in some idiosyncratic fashion. That is, they want to consider both content mastery and performance relative to peers in assigning grade cutoffs.

One very subjective combination method is to examine the distribution of test scores. Using what is commonly called the "eyeball method," instructors draw lines through various points of the distribution to represent grade cutoffs. The procedure is basically arbitrary. If pressed to give a reason why a particular cutoff point was selected, an instructor may reply that "it looked about right." Others select cutoff points where they see a gap in the distribution of student scores. Gordon (1988) notes "It is my experience that the ideal grading technique, both fair and motivational ... is 'cluster grading'"' (p. 1). He notes:

Here is one such actual distribution: $949492 * 898988$ $87 * 84 * 8281808079 * 777775747373737271$ $707069 * 6665 * 6362 * 6059595454 * 5251 * 47$ 46. Note that 10 clusters appear. It remains only to assign grades to these clusters-A+, A, A-, B+, B, B-, and so on.... It has been my experience that students enjoy this type of grading, since they feel they are being evaluated with respect to their peers in a fair and competitive manner. (p. 1)

Many instructors might reject this method as unacceptably arbitrary. However, instructors who believe that strict reliance on either content mastery or peer comparison grading is unacceptable are left to attempt to combine the two approaches on their own. For example, they may arrange students' scores in an overall rank ordering, noting both the percentage correct and percentile scores of each student. Using the eyeball method, they draw grade cutoff 
lines at various scores on the basis of some subjective combination of the percentage correct and percentile scores.

Although the attempt to combine a consideration of content mastery and performance relative to peers may be commendable, the combination methods described above are still problematic. The most glaring problem is that the selection of cutoff points is still left to intuition. As a consequence, the inconsistency and variation in grade distributions among faculty members remains. There is still room for grade inflation, and students may eventually identify "hard" and "easy" graders among the faculty.

Faculty who prefer a combinatorial approach to grading (i.e., a synthesis of content mastery and peer comparison methods) need a standard method to use to combine the absolute measure (percentage correct) and the comparative measure (percentile score). Various such combination procedures have been proposed (Aiken, 1983; Ebel, 1979; Lein, 1980). These methods are rather complicated, however, and they require that instructors compare the performance on past and current courses. A problem with these methods is that the difficulty level of courses may change over time, particularly given the introduction of a new textbook or teaching method.

\section{GRADER: A PROGRAM TO STANDARDIZE LETTER GRADE CUTOFFS}

GRADER is an IBM-PC/AT-compatible computer program that makes student test and/or course letter grade recommendations to instructors. The program uses an algorithm to combine the content mastery (percentage correct) and peer comparison ("curve") approaches to set grade cutoffs. In addition to standardizing the procedures with which letter grade cutoffs are made, the use of GRADER may help to reduce instructor grading subjectivity and obviate tendencies toward grade inflation or deflation.

\section{Data Input}

To use GRADER, instructors simply create an ASCII file of student test and/or total course points with their word processing software. In this input file, each line has each student's name or ID, followed by the student's test and/or course points. At the user's request, the program will sum all test points for each student.

\section{Calculation of Student Letter Grades}

GRADER bases its grade assignment recommendations on a consideration of both percentage correct and percentile scores.

There are at least two methods of calculating percentile scores (Lyman, 1986). With a distribution of, say, 50 scores, the lowest percentile according to one method of calculating percentiles would be 1 . With another method of calculating percentiles, the lowest percentile would be higher, perhaps 7 . The latter method of producing percentiles is used by GRADER, because it does not overly penalize students with low scores in small classes.

GRADER calculates two grades for each student by using the typical cutoff points of content mastery grading and peer comparison grading, respectively (see Appendix $B$ for the exact cutoffs). These two grades are then converted to index numbers on a 13-point scale that corresponds to the grade range (e.g., $\mathrm{F}=1, \mathrm{~F}+=2, \mathrm{D}-=$ $3, \ldots \mathrm{A}-=12, \mathrm{~A}=13$ ). The average of these two index numbers is calculated. The grade associated with the resulting average is the student's grade. For example, if a student's percentage correct score was 80 (which would be a $\mathrm{B}-$, using the traditional percentage correct cutoffs) the student's "content mastery" score would be 9 . If the student's corresponding percentile score was 93 (which would be an A-, using the standard grading curve cutoffs noted earlier), the "peer comparison" score for the student would be 12 . The combined score (taking the average of 9 and 12) would be 10.5 , rounded up to 11 . Thus, GRADER would recommend a letter grade of B+.

Although the selection of letter grade cutoffs (noted in Appendix B) for the content mastery and peer comparison grades is arguably arbitrary, with the use of GRADER the process of arriving at letter grades is (1) standardized and (2) based on an evaluation of both content mastery and peer comparison data. GRADER does allow instructors to change either the content mastery or the peer comparison cutoff, or both. However, once the grade cutoffs have been set, it subverts one purpose of this procedure (standardization) to arbitrarily change them on a frequent basis.

\section{Possible Advantages of Using GRADER}

GRADER is fundamentally a means of standardizing grade assignment practices in a way that takes into account both content mastery and peer comparison information. In addition, the use of GRADER offers the possibilities of reducing grading subjectivity, achieving greater grading consistency among the faculty and greater grade comparability between different courses, and the reduction of grade inflation (or deflation). Moreover, students are evaluated in terms of their performance in comparison with their peers, as well as their demonstrated degree of mastery of the course content.

One way to illustrate this is to examine how GRADER, in comparison with either content mastery or curve grading methods used alone, deals with extreme situations. For example, suppose all the students in a class got $100 \%$ correct on a test. It could mean that all of the students had mastered all of the material. More likely, it suggests that the test was far too easy-that the "test ceiling" was too low. There probably are significant, measurable differences in knowledge between the various students, which the test, due to its low ceiling, was unable to reflect. Thus, more likely, all students probably do not deserve 
an A. However, traditional content mastery grade cutoffs would in fact grant all the students an A.

In contrast, traditional peer comparison grading would give all the students a $\mathrm{C}$. This grade might be too low, and students would likely perceive it as unfair.

With the use of GRADER, all students in the class would receive a $B$ on the test-a middle ground between the grades produced by content mastery and peer comparison cutoff methods.

Another advantage of using GRADER is that, because both absolute and comparative information are used to determine grade cutoffs in a standardized fashion, the grades produced by the various faculty members who use GRADER are more comparable. Tendencies toward grade inflation can be reduced significantly. Bickering over grades is also reduced, because students unhappy with their grades would have a difficult time arguing their cases, given their percentile and percentage correct scores, as well as their understanding of the grade cutoff system at the outset of the course. Instructors who use GRADER cannot be accused of unfairness in making grade cutoffs, since they no longer make arbitrary grade cutoff decisions (although they may still be accused of making unfair tests).

\section{Program Availability}

GRADER, and sample handouts for students describing the grading method, are available from the author to anyone who sends a formatted IBM-PC-compatible 3.5-in. disk in a returnable mailer.

\section{REFERENCES}

AIKRN, L. R. (1983). Determining grade boundaries on classroom tests. Educational \& Psychological Measurement, 43, 759-763.

Bauernfeind, R. H. (1962). The matter of "ipsative" scores. Personality \& Guidance Journal, 41, 210-217.

Bresee, C. W. (1976). On grading on the curve. Clearing House, 50 , 108-110.

CuRETon, L. E. (1971). The history of grading practices. NCME Measurement in Education, 2, 1-8.

Dressel, P. (1983). Grades: One more tilt at the windmill. In A. W. Chickering (Ed.), Bulletin. Memphis, TN: Memphis State University, Center for the Study of Higher Education.

EBeL, R. L. (1979). Essentials of psychological measurement (3rd ed). Englewood Cliffs, NJ: Prentice-Hall.

GoRDoN, L. (1988, April). Cost-benefit testing. Academic Leader, 4(4), 1-2.

HanNa, G. S., CaShiN, W. E. (1988). Improving college grading (Idea Paper No. 19). Manhattan, KS: Kansas State University, Center for Faculty Evaluation and Development.

Hicks, L. E. (1970). Some properties of ipsative, normative, and forced-choice normative measures. Psychological Bulletin, 74, 167-184.

Kaplan, R. M., \& Saccuzzo, D. P. (1982). Psychological testing: Principles, applications, and issues. Monterey, CA: Brooks/Cole.

LEIN, A. J. (1980). Measurement and evaluation of learning (4th ed.). Dubuque, LA: Brown.

Lyman, H. B. (1986). Test scores and what they mean (4th ed.). Englewood Cliffs, NJ: Prentice-Hall.

Popham, W. J. (1978). Criterion-referenced measurement. Englewood Cliffs, NJ: Education Technology Publications.

\section{APPENDIX A \\ Sample Score Distribution and GRADER Grade Recommendations}

The following table contains a hypothetical distribution of student test or course points (with associated percentage correct and percentile scores) and GRADER-recommended grade assignments. Readers may wish to cover the right side of the table (from the Content Mastery Grade column to the right), and make their own grade assignments to compare their own subjective method of making grade cutoffs with the grade assignments produced by GRADER.

GRADER Generated Grade Assignments

\begin{tabular}{lcccccc}
\hline & \multicolumn{5}{c}{ Content } & \multicolumn{3}{c}{ Pear } \\
Name & Score & Correct & Grade & Score & Grade & Grade \\
\hline 5065 & 166 & 94 & A & 98 & A & A \\
6121 & 164 & 93 & A & 95 & A & A \\
3685 & 159 & 90 & A- & 91 & A & A \\
3424 & 159 & 90 & A- & 91 & A & A \\
8248 & 158 & 90 & A- & 86 & A- & A- \\
6023 & 158 & 90 & A- & 86 & A- & A- \\
8184 & 155 & 88 & B+ & 81 & B+ & B+ \\
0488 & 151 & 86 & B & 79 & B & B \\
5204 & 150 & 85 & B & 75 & B & B \\
4524 & 150 & 85 & B & 75 & B & B \\
7548 & 148 & 84 & B & 69 & B- & B \\
4723 & 148 & 84 & B & 69 & B- & B \\
9679 & 147 & 84 & B & 63 & B- & B \\
2778 & 147 & 84 & B & 63 & B- & B \\
2434 & 146 & 83 & B & 59 & C+ & B- \\
8380 & 144 & 82 & B- & 55 & C+ & B- \\
0307 & 144 & 82 & B- & 55 & C+ & B- \\
6260 & 143 & 81 & B- & 51 & C+ & B- \\
0285 & 142 & 81 & B- & 48 & C & C+ \\
8688 & 141 & 80 & B- & 45 & C & C+ \\
8946 & 139 & 79 & C+ & 43 & C & C+ \\
9189 & 138 & 78 & C+ & 38 & C & C+ \\
8404 & 138 & 78 & C+ & 38 & C & C+ \\
7959 & 137 & 78 & C+ & 33 & C & C+ \\
4242 & 137 & 78 & C+ & 33 & C & C+ \\
$\mathbf{0 0 6 3 3}$ & 134 & 76 & C & 29 & C- & C \\
9751 & 133 & 76 & C & 23 & C- & C \\
9081 & 133 & 76 & C & 23 & C- & C \\
3827 & 133 & 76 & C & 23 & C- & C \\
9225 & 131 & 74 & C & 15 & D+ & C- \\
2704 & 131 & 74 & C & 15 & D+ & C- \\
2580 & 131 & 74 & C & 15 & D+ & C- \\
1643 & 128 & 73 & C & 8 & D- & D+ \\
0814 & 128 & 73 & C & 8 & D- & D+ \\
8189 & 126 & 72 & C- & 4 & F+ & D \\
4091 & 122 & 69 & D+ & 1 & F & D-
\end{tabular}

Number of:

As $616 \%$

Bs $12 \quad 33 \%$

Cs $14 \quad 38 \%$

Ds $411 \%$

Fs $\quad 0 \quad 0 \%$

$M=142.750 \quad S D=11.136$ 


\section{APPENDIX B \\ Sample Student Handout Describing the GRADER Grading System}

Your grades on tests, and in the class overall, will be determined by the average of two grades: a "content mastery grade" and a "peer comparison grade." The first is based on your percentage correct score on the test; the second is based on your percentile score (the percentage of your classmates who had a score lower than yours).

A. Content mastery grade. This grade will be determined by your percentage correct score.

$\begin{array}{ll}\text { A } \geq 93 & \text { A- }=90-92 \\ \text { B }=87-89 & \text { B }=83-86 \\ \text { C }=77-79 & \text { C }=73-76 \\ \text { D+ }=67-69 & \text { D }=63-66 \\ \text { F+ }=57-59 & \text { F }<57\end{array}$

$\mathrm{B}-=80-82$

$\mathrm{C}-=70-72$

D- $=60-62$

B. Peer comparison grade. This grade will be determined by how well you perform in relation to your peers, as indicated by your percentile score (the percentage of students in the class with a score lower than yours).

Grade Percentile

$\begin{array}{lll}\text { A } \geq 90 \% \text { ile } & \text { A- }=85-89 \\ \text { B }=80-84 & \text { B }=70-79 \\ \text { C }=50-59 & \text { C }=30-50 \\ \text { D+ }=15-19 & \text { D }=10-14 \\ \text { F+ }=4-6 & \text { F }<4\end{array}$

$B-=60-69$

C $-=20-29$

$\mathrm{D}-=7-9$
C. Conversion of grades to a 13-point scale. The above two grades will be converted to a numerical grade index on a 13 . point scale: $F=1, F+=2, D-=3, D=4, D+=5, C-=$ $6, C=7, C+=8, B-=9, B=10, B+=11, A-=12$, $\mathrm{A}=13$.

D. The numbers that correspond to your two grades will be averaged. Your grade is the grade that corresponds to the resulting average.

For example, suppose that you took a very difficult test and got only $69 \%$ of the items correct. You might be very concerned about your grade (and, indeed, if only percent correct were taken into consideration, your grade would be a D in many traditionally graded classes). But imagine that your peers also found this test to be very difficult, and that you did relatively well when compared with them. Say that your score was better than $85 \%$ of the class scores on the test.

To calculate your grade, you would note that your "content mastery" grade (based on your $69 \%$ correct score) is a C-. and that your "peer comparison" grade (based on your percentile score of 85) is an A-. These grades correspond to numerical grade indices of 6 and 12 , respectively. The average of 6 and 12 is 9 . A score of 9 corresponds to a grade of B-. Therefore, your grade on the test is a B-.

You may wish to plug in different values in the equation (you will find that things are a bit tougher if you perform poorly on a test that your peers have found easy!). This method takes into account both your mastery of the material and the relative ease or difficulty of the test. In comparison with other grading methods, I think that you will find that this is a very a fair way to assign grades. 